\title{
The relationship between the implementation and effectiveness of a nationwide physical activity telephone counseling service for adults with spinal cord injury
}

Jennifer R. Tomasone ${ }^{1 \S}$, Kelly P. Arbour-Nicitopoulos ${ }^{2}$, Amy E. Latimer-Cheung ${ }^{1}$, \& Kathleen A. Martin Ginis ${ }^{3}$

${ }^{1}$ School of Kinesiology \& Health Studies, Queen's University, Kingston, Canada

${ }^{2}$ Faculty of Kinesiology \& Physical Education, University of Toronto, Toronto, Canada

${ }^{3}$ School of Health and Exercise Sciences, University of British Columbia Okanagan, Kelowna, Canada

\author{
${ }^{\S}$ Corresponding author: \\ Jennifer R. Tomasone \\ School of Kinesiology and Health Studies, Queen's University \\ 28 Division St., Kingston, Ontario, Canada, K7L 3N6 \\ Phone $+01-613-533-6000 \times 79193$ \\ Fax $+01-613-533-2009$ \\ E-mail tomasone@queensu.ca
}

Keywords: knowledge translation, Knowledge-to-Action framework, leisure-time physical activity, exercise, physical disability 


\section{Abstract}

Purpose: Get in Motion (GIM) is an evidence-based telephone counseling service that promotes leisure-time physical activity (LTPA) among Canadian adults with spinal cord injury (SCI). The first phase of GIM sustained intentions for, and increased participation in, LTPA; however, it is unclear how GIM led to these outcomes. The purpose of this study was to explore the implementation correlates of change in LTPA intentions and behaviour in the second phase of GIM.

Method: The frequency, duration, and content of counseling sessions were tailored to meet clients' $(N=46 ; 50.0 \%$ male; $50.0 \%$ paraplegia; 51.46 (SD 12.36) years old) needs and preferences. Intervention dose and content were monitored using Counseling Session Checklists. Clients self-reported their intentions for and actual aerobic and strength-training LTPA participation at baseline, 2-, 4-, and 6-months, and their perceptions of service quality at 6months.

Results: The second phase of GIM effectively sustained LTPA intentions and increased time spent on moderate-to-vigorous strength-training and total LTPA. Increases in clients' moderateto-vigorous aerobic LTPA were significantly positively related to intervention dose, intervention content (both informational and behavioural strategies), and clients' perceptions of service credibility.

Conclusions: This study identified intervention dose and content as key implementation variables for an LTPA telephone counseling service for adults with SCI. 


\section{Introduction}

Among people with spinal cord injury (SCI), engaging in leisure-time physical activity (LTPA) - physical activity that one chooses to engage in one's spare time, such as exercising or playing sports [1] - has been shown to impart physical [2, 3], psychological [4], social [5], and quality of life benefits [6,7]. Despite accumulating research evidence about these benefits, approximately $50 \%$ of adults with SCI do not engage in LTPA [8]. A commonly reported barrier to LTPA participation is a lack of resources and services that translate LTPA research evidence to this group [9]; thus, the implementation of evidence-based LTPA-enhancing information, programs, and services in the SCI community remains a priority.

\section{Knowledge-to-Action (KTA) Framework}

The Knowledge-to-Action (KTA) framework [10] provides a conceptual approach that is useful for implementing research evidence in a practical setting. This framework is divided into two processes - knowledge creation and knowledge application (action cycle) - that can influence one another throughout the KTA process; that is, emerging research evidence can lead to refinements in the action cycle, and findings from the action cycle can lead to the generation of new evidence [10].

\section{Knowledge Creation of LTPA Interventions}

Examples of knowledge tools and products that can be derived from the process of knowledge creation are evidence-based resources or interventions that target theory-based determinants of LTPA [11]. For example, knowledge products such as the Physical Activity Guidelines for Adults with SCI [12], the SCI Get Fit Toolkit [13], and Active Homes manuals and videos [14] have been developed to provide people with SCI with recommendations for how much LTPA to do, information about the benefits of and opportunities for LTPA, and suggestions 
for engaging in home-based strength-training LTPA, respectively. While effective at altering theory-based determinants of behaviour (e.g., attitudes, self-efficacy, perceived behavioural control; e.g., [15]), LTPA informational interventions are not particularly effective at changing actual LTPA behaviour. This is true in both the general [16] and SCI populations [17, 18]. However, informational interventions may be effective when combined with behavioural intervention approaches that teach people skills to initiate and maintain LTPA participation.

Two randomized controlled trials (RCTs) have examined the efficacy of providing people with informational resources and then teaching them theory-based behavioural strategies to increase their LTPA $[19,20]$. All participants in both RCTs first received the same LTPA information (e.g., basic LTPA instruction guide and a pamphlet with tips for initiating an LTPA routine). The experimental group in the first RCT, who used the behavioural skill of action planning alone, sustained their LTPA intentions, increased their confidence to schedule LTPA, and increased their LTPA behaviour compared to participants who only received the informational resources [20]. The second RCT compared two groups of participants who were taught to engage in either action planning alone or action and coping planning combined [19]. While both groups increased their LTPA behaviour, participants who were taught to create both action and coping plans reported significantly greater confidence to schedule LTPA and overcome LTPA-related barriers, as well as greater LTPA behaviour, than participants who were only taught to form action plans. These results suggest that teaching people with SCI a greater number of behavioural strategies in a single intervention may lead to greater increases in both theory-based determinants of and actual LTPA participation.

\section{Action Cycle of LTPA Interventions}

LTPA-enhancing interventions do not achieve their full potential if they are not taken out of knowledge creation and put into action beyond the original research context in which they 
were developed [21-23]. In June 2008, the two RCTs described above [19, 20] were translated into Get in Motion (GIM), a nationwide LTPA telephone counseling service that is offered at no cost to Canadian adults with SCI. GIM is an evidence- and theory-based knowledge service designed to provide LTPA information (e.g., [12-14]) and to teach behavioural skills [19, 20] shown to be efficacious for enhancing theory-based determinants of LTPA participation in the SCI community [24, 25]. From its inception in June 2008 to June 2011, GIM was implemented as a real-world service across Canada [26]. Our preliminary evaluation showed that clients' LTPA intentions were sustained throughout the service, and that more clients were active at the end of the service than at the beginning. These results suggest that GIM is effective at promoting LTPA intentions and behaviour in the SCI community; however, questions remain as to how the service leads to these outcomes. The present study builds upon this previous work [26] by striving to answer these implementation-specific questions.

\section{Where One Action Cycle Ends, Another Begins}

Because GIM is implemented under "real-world conditions", the frequency and duration of counseling sessions are adjusted to accommodate individual clients' needs and schedules, resulting in some clients receiving greater exposure to the service than others. Further, the counselor tailors the discussion of informational and behavioural strategies to individual client interests, motivations, and/or barriers to LTPA. Moreover, clients' perceptions of the quality of GIM may influence their level of participation in the service. Thus, intervention dose, content, and perceived quality can vary among clients. Each of these implementation variables reflects how GIM is used in practice, and may be "key ingredients" that influence the service's effectiveness $[27,28]$. Understanding the relationship between these variables and the service's effectiveness in a real-world setting would help to identify how the implementation of GIM impacts LTPA-related outcomes among clients, and inform future refinements of the service [28]. 
Intervention dose, content and perceived quality may be potential mechanisms by which an LTPA-enhancing intervention achieves its outcomes [29]. In the broader LTPA telephone counseling literature, a positive relationship between intervention dose and behavioural outcomes has been established [30]. For example, a previous study of a community-based telephone counseling service for adults with Type 2 diabetes and hypertension found a positive association between intervention dose and multiple behavioural outcomes (LTPA, fat intake, fibre intake; [31]); however, the relationship between counseling session content and the service's effectiveness was not investigated. While reviews have identified positive relationships between intervention content (e.g., behaviour change techniques) and outcomes in controlled efficacy studies [32, 33], additional research is needed to understand how intervention content and quality influence the effectiveness of LTPA-enhancing interventions in an applied setting. Research examining these variables requires rigorous monitoring of implementation throughout the intervention; the present study addressed this gap in the literature.

\section{Objectives}

The purpose of this study was to explore the implementation correlates of change in LTPA intentions and behaviour among GIM clients who enrolled in the second phase of the GIM service between September 2011 to January 2014. During this phase, an enhanced monitoring and evaluation protocol was implemented so that questions regarding the relationship between implementation and effectiveness could be examined.

To address our research questions, we first examined whether clients' LTPA intentions and behaviour changed throughout their enrollment in the service. Based on our previous research $[19,20,26]$, we hypothesized that over their 6-month enrolment in GIM, clients would report (a) strong intentions at baseline that would be sustained throughout enrolment, and (b) an increase in moderate-vigorous intensity LTPA. Second, we explored whether the following 
implementation variables were related to changes in clients' LTPA intentions and behaviour: (1) dose (i.e., the number and duration of counseling sessions), (2) content (i.e., number of times and duration of discussion of informational vs. behavioural strategies), and (3) clients' perceived quality of the service. Based on the findings from community-based telephone counseling interventions in other populations [30], we hypothesized that intervention dose would be related to increases in LTPA intentions and behaviour. In the absence of previous LTPA implementation literature examining the influence of real-world intervention content and perceived quality on effectiveness, no hypotheses were put forth for the relationship between these variables and changes in LTPA intentions and behaviour.

\section{Method}

\section{Procedure}

The GIM service and data collection procedures were approved by the McMaster University Research Ethics Board.

Participants. Between September 2011 and January 2014, GIM was advertised through the SCI Action Canada website [34], LTPA-related community events hosted by SCI Action Canada or their partner organizations, articles written for SCI-related publications (e.g., SCI Ontario's Outspoken magazine) and websites (e.g., PAR-QoL website), and word of mouth. Promotion and enrollment of clients were ongoing, and 46 clients enrolled in GIM during this period. These clients had a mean age of 51.46 years (SD 12.36) and were injured on average 17.00 years (SD 17.59) prior to their enrollment in the service. Half of the clients were male, had paraplegia, and used a power wheelchair as their primary mode of mobility outside their home (all $n \mathrm{~s}=23,50.0 \%$ ). The majority of clients had obtained post-secondary education (college, 
undergraduate or graduate degree, or professional program; $n=31,67.4 \%)$, were married $(n=$ 24, 52.5\%), and were from Central Canada (Ontario or Quebec; $n=25 ; 54.3 \%$ ). The most commonly reported causes of injury were sports/recreation/falls $(n=13,28.2 \%)$ and motor vehicle accidents $(n=12,26.1 \%)$. See table 1 for complete demographic information for the clients. Overall, the 46 clients were representative of the Canadian adult SCI population on all measured demographic variables except for sex, primary mode of mobility, and geographic location [35-37]. Compared to the Canadian SCI population, a greater proportion of the GIM clients were females (50\% vs. $19 \%$ of Canadian SCI population), power wheelchair users $(50.0 \%$ vs. $23.0 \%$ of Canadian SCI population), and residents from Central Canada (54.3\% vs. $28.0 \%$ of the Canadian SCI population).

\section{Insert table 1 about here}

Enrollment procedures. Interested potential clients contacted the GIM Program Coordinator directly via postal mail, e-mail, or a toll-free telephone number. During a phone interview, the Coordinator assessed individuals' eligibility to participate in the service (i.e., currently residing in Canada, at least 18 years of age, traumatic or non-traumatic SCI) and provided eligible clients with more information about the enrollment procedures and the protocol of the 6-month service. Individuals were given the opportunity to ask questions about the service before enrolling. All clients who enrolled provided informed consent for the Coordinator to collect demographic information and for the counselor to keep a record of counseling sessions. After collecting demographic information, the Coordinator mailed a Welcome Package to the client which included the Physical Activity Guidelines for Adults with SCI [12], the SCI Get Fit Toolkit [13], two elastic resistance bands (Thera-Band $®)$ and instruction guide, an exercise safety tip sheet, tips and strategies for meeting LTPA goals, and an activity intensity classification chart [38]. The client's first (baseline) counseling session was scheduled by the 
Coordinator to be approximately two weeks from the date of enrollment to ensure that the client would have time to review the Welcome Package prior to speaking with the counselor.

Clients were then invited to participate in an ongoing quality evaluation study of the GIM service. Those interested in completing additional questionnaires prior to and at 2-, 4-, and 6months after their first counseling sessions provided verbal consent and selected whether they would like to have their questionnaires administered via a telephone interview or electronically through Fluid Surveys. A research assistant then took over the data collection procedures for clients who enrolled in the evaluation study.

Telephone-based counseling. All counseling sessions were delivered over the phone by a single exercise counselor who was a registered Kinesiologist and had extensive experience in developing and implementing community-based LTPA programs for persons with SCI. Specific to GIM, the counselor initially received professional training in motivational interviewing and behaviour change theories, then met monthly with members of the research team (JRT and KAN) to support and troubleshoot implementation, as well as to discuss emerging evidence and theory about LTPA participation among adults with SCI.

Counseling sessions were scheduled at a mutually-convenient time for the client and counselor. The standard protocol for counseling session frequency was weekly for the first two months, biweekly for months 2 to 4 , and then monthly for months 4 to 6 in order to gradually transition clients from dependency on the counselor to independent self-regulation of LTPA behaviour [39]. However, clients were given the opportunity to self-select this standard frequency or another frequency that met their individual needs, interests and schedule.

The intervention strategies used during the counseling sessions can be classified as either informational or behavioural ([16]; refer to table 2 for a list of strategies and how they are used in GIM). The counselor tailored the strategies discussed to meet each individual client's LTPA 
interests, motivations and barriers. For example, in a given session, if a client expressed uncertainty about how much LTPA s/he should be doing or how to do it safely, then the counselor focused on providing this information. If a client expressed difficulty scheduling LTPA into their week or overcoming barriers, then the counselor directed the conversation towards teaching behavioural strategies. The duration of each counseling session was dependent on the number of topics that were discussed as well as the number of questions that the client had about a given strategy. To keep track of content and duration for each session a client received, the counselor completed a Counseling Session Checklist (described in the next section).

\section{Insert table 2 about here}

If a counseling session fell within a two-week period of 2-, 4-, or 6-months since a client's first session, the LTPA behaviour measure was administered at the beginning of the session in order to: (1) provide the counselor with feedback about a client's current LTPA behaviour, which could then be used to tailor the remaining content of the session, and (2) ensure that LTPA participation from all clients enrolled in the service could be tracked. Clients could choose to discontinue the service at any time. Clients who completed their 6-month enrollment were contacted by the Program Coordinator after their last session to complete a Client Reflection.

\section{Measures}

LTPA intentions. If clients volunteered to participate in the ongoing quality evaluation study of GIM (discussed above), their intentions to engage in LTPA were assessed at baseline (before their first counseling session), as well as at 2-, 4-, and 6-months after enrollment. Two items followed the stem, "In the next two months, I intend to engage in...": (1) “At least 20 minutes of moderate-to-vigorous aerobic activity on at least two days of the week", and (2) "At least 3 sets of 8-10 repetitions of moderate-to-vigorous strength-training activity for each muscle 
group at least two days per week". These items were used in previous studies examining LTPA intentions in the SCI community [19, 20, 26, 40], but were modified to reflect clients' intentions to meet both the aerobic and strength-training recommendations outlined in the Physical Activity Guidelines for Adults with SCI [12]. Clients were asked to rate their level of agreement with the two items on a 7-point Likert-type scale $(1=$ strongly disagree, $7=$ strongly agree $)$, with higher values indicating greater intentions to engage in moderate-to-vigorous LTPA. The two items were analyzed separately, as well as averaged to give an overall intention measure $(r \mathrm{~s} \geq .59)$.

LTPA behaviour. The 7-day, self-report LTPA Questionnaire for People with SCI [41] was used to assess clients' LTPA behaviour. The LTPA Questionnaire for People with SCI has shown acceptable test-retest reliability and construct validity in previous research [42]; however, this measure was modified in the current study to assess both aerobic activity and strengthtraining activity, in order to correspond with the LTPA recommendations outlined by the Physical Activity Guidelines for Adults with SCI [12]. Clients were read the definitions of mild-, moderate-, and vigorous-intensity aerobic LTPA and recalled the number of days, over the past seven days, that they performed aerobic LTPA at each intensity. They were then asked to recall how many minutes they usually spent doing aerobic LTPA at that intensity each day. The aerobic scale was scored by calculating the total number of minutes of aerobic activity performed at each intensity (number of days of aerobic activity x number of minutes of aerobic activity each day) over the past week. Minutes of moderate- and vigorous-intensity aerobic activity were summed to give a total number of minutes of aerobic moderate-vigorous physical activity (MVPA) performed in a given week. The same process was completed for strength-training MVPA performed in a given week. Total MVPA for the week was calculated by summing aerobic and strength-training MVPA. For the current study, only moderate-vigorous LTPA was 
examined as activity at these intensities are required for people with SCI to achieve fitness benefits [2].

Counseling Session Checklist. The Counseling Session Checklist served as: (1) a roadmap for the counselor to follow during the session, (2) a record of clients' needs, interests, and preferences with respect to their motivation for participating in both LTPA and the service, and (3) a tool to collect data about the implementation of the service (i.e., type and duration of strategies discussed with the client during each session). To ensure the Checklist could be adopted and implemented in practice [43], the counselor was involved in the creation, pilot testing, and refinement of the Checklist.

To address our research question, information about six different implementation variables was extracted from the Checklists. Two variables assessed intervention dose: (1) number of sessions the client received and (2) duration of each session. Four variables assessed intervention content: (1) number of times informational strategies were discussed, (2) time spent discussing informational strategies, (3) number of times behavioural strategies were discussed, and (4) time spent discussing behavioural strategies. To track time spent on a strategy, the counselor indicated the percentage of total call duration that was spent on a given strategy, which was then converted to minutes (i.e., percentage of total call duration spent on strategy $\mathrm{x}$ total call duration $=$ time spent on strategy). The total number of times and time spent on both informational and behavioural strategies were summed for each session, and then summed across each two-month period of counseling.

The first author received permission from five different clients at different points in the service to listen in on a counseling session (five sessions total) and completed a reliability check of counselor-completed Checklists for these sessions. The level of agreement between the 
researcher and counselor for strategies discussed was $100 \%$, and intraclass correlations for the percentage of session duration spent on given strategies were $\geq .987$ across the five sessions.

Client Reflection. To assess perceived quality of implementation, clients were asked to rate the following five items on a 7-point Likert-type scale $(1=$ strongly disagree, $7=$ strongly agree): "The information and resources that the counselor provided were: (1) interesting; (2) easy to understand; (3) credible; and (4) personally important to me"; and (5) "I learned a lot of new information from the GIM counselor".

\section{Statistical Analysis}

Data were screened for outliers and missing data using established guidelines [44]. Three LTPA outliers (values $>3$ SDs above the mean) were identified (one for baseline aerobic MVPA, one for baseline strength-training MVPA, and one for 6-month aerobic MVPA; each outlier was reported by a different client) and adjusted to a value that was exactly $3 S D$ s above the mean. Due to the large number of clients who discontinued their enrollment in the service between baseline and 2 months (see figure 1), 11 (31.4\%) clients had data for the intentions measure and $16(38.1 \%)$ clients had data for the behaviour measure at only one time point (i.e., baseline), ruling out the use of missing value imputation techniques as there was a moderate amount of missing data from a relatively small sample size [44]. Intent-to-treat analysis was not used for the implementation data given that the underlying assumption of no change would not be appropriate for the clients who discontinued their enrolment and therefore would have also received the fewest calls. Conducting an intent-to-treat analysis would over-estimate associations between calls completed and outcomes.

Based on the standard GIM session frequency (weekly for the first two months, biweekly for months 2 to 4 , and then monthly for months 4 to 6 ), the number of counseling sessions should be greatest between baseline and 2-months (eight sessions) and lowest between 4-6 months (two 
sessions). Indeed, an ANOVA revealed that the average number of counseling sessions that the clients actually received between baseline to 2 months was significantly greater than the average number of counseling sessions received between both months 2 to 4 and 4 to 6 ( $p s<.001)$. No significant differences between the number of conselling sessions emerged between months 2 to 4 and 4 to $6(p=.39)$. To minimize loss of data in subsequent paired comparisons, the 2 to 4 month and 4- to 6-month counseling periods were collapsed into a single 2 to 6-month period.

Descriptive statistics were calculated for demographic variables, LTPA intentions (aerobic, strength-training, and mean), LTPA behaviour (aerobic, strength-training, and total MVPA), the six implementation variables from the Counseling Session Checklists, and Client Reflection variables. To examine changes in LTPA intentions and behaviour, separate repeated measures ANOVAs were performed, and significant ANOVAs were followed up by post-hoc paired $t$-tests to determine where significant changes occurred. Cohen's $d$ was calculated as an index of effect size for changes in LTPA intentions and behaviour between time points. Effect sizes were interpreted as small, medium, and large-sized effects using Cohen's conventions of $d$ s of $0.20,0.50,0.80$, respectively [45]. To examine the correlates of change in LTPA intentions and behaviour, change scores were calculated for aerobic, strength-training and mean/total intentions and MVPA between baseline to 2 months, 2 to 6 months, and baseline to 6 months. Bivariate correlations were then calculated between change scores and measures of implementation dose, content, and perceived quality.

\section{Results}

\section{Client Flow}


Four clients discontinued the service before their baseline counseling session. Of the 42 clients who received counseling, 25 completed all 6 months of counseling, with the greatest number of clients discontinuing participation between baseline and 2 months $(n=13)$. Figure 1 shows the number of clients and the data available for these clients at baseline, 2-, 4-, and 6months. Demographic characteristics of (a) clients who dropped out between baseline and 2 months $(n=13)$ versus clients who continued on to months 2 to 6 of service $(n=29)$, and (b) clients who dropped out at any point between baseline and 6 months $(n=21)$ versus clients who completed the 6 month service ( $n=25$ ) were compared using independent samples $t$-tests for continuous variables, and chi-square analysis for dichotomized variables (see table 1). No significant demographic differences between these groups of clients emerged (all $p \mathrm{~s} \geq .10$ ).

\section{Insert figure 1 about here}

\section{LTPA Intentions}

Because the LTPA intention measure was administered by a research assistant as part of the ongoing quality evaluation study, and not all clients were interested in completing these additional questionnaires, the sample size for the intention measure at a given time point is less than the number of clients enrolled in the service (see figure 1).

As hypothesized, clients' baseline intentions for engaging in aerobic, strength-training, and total LTPA were high $(M(S D) \geq 6.14$ (1.44), out of 7) and did not change over the course of the 6-month service (all $F \mathrm{~s}(2,30) \geq 0.690, p \mathrm{~s} \geq .24)$. Table 3 lists all descriptive statistics, $F$ statistics and Cohen's $d$ for LTPA intentions over the 6-month period.

Insert table 3 about here

\section{LTPA Behaviour}


Significant time effects were seen for changes in time spent in strength-training and total MVPA over the 6-month period (all $F(2,40) \mathrm{s} \geq 3.679, p \mathrm{~s} \leq .03)$. Post-hoc paired sample $t$-tests revealed significant increases in time spent in strength-training and total MVPA between baseline and 2 months $(d \mathrm{~s} \geq .30)$ and between baseline and 6 months ( $p \mathrm{~s} \leq .017 ; d \mathrm{~s} \geq .43)$. No significant changes in time spent in strength-training or total MVPA were seen between 2 to 6 months ( $p$ s $\geq .23$ ). No significant changes in aerobic MVPA emerged over the 6 month period; however, small effect sizes for increases in aerobic MVPA emerged between baseline to 2 months $(d=.29)$ and between baseline to 6 months $(d=.20)$.

\section{Implementation Correlates of Change in LTPA Intentions and Behaviour}

Measures of dose and content. Regarding dose, during the first two months of the service, GIM clients had an average of 3.22 (SD 1.98) counseling sessions and an average session duration of 58.39 minutes (SD 36.98). Regarding the content discussed over the first two months, informational strategies were discussed 7.35 times (SD 6.88) for an average total of 17.31 minutes (SD 17.33), while behavioural strategies were discussed 5.96 times (SD 4.09) for an average total of 17.50 minutes (SD 14.91). The means for all measures of implementation dose and content were greater between baseline to 2 months than 2 to 6 months ( $p s \leq .02$ ). Table 4 provides descriptive statistics for the measures of dose and content during the service.

\section{Insert table 4 about here}

When examining the type of content that was discussed, paired samples $t$-tests revealed that there were no significant differences between time spent discussing informational versus behavioural strategies between baseline to 2 months and 2 to 6 months. No significant differences were found between the number of times that informational and behavioural strategies were discussed between baseline to 2 months; however, informational strategies were 
discussed significantly more times than behavioural strategies between 2 to 6 months $(t(45)=$ $3.815, p<.001)$.

Changes in aerobic MVPA between baseline to 6 months were significantly related to total session duration, total number of sessions, and the number of times that informational and behavioural strategies were discussed over the 6-month period $(.398 \leq r \mathrm{~s} \leq .534$; $p \mathrm{~s}<.05$; see table 5). Clients reporting the greatest changes in aerobic MVPA over the 6-month service also had the greatest intervention dose, and discussed a greater number of both informational and behavioural strategies with the counselor. No other correlations were statistically significant.

Measures of intervention dose and content were also significantly related $(p s<.01$; see table 5). A mediation model was examined to determine whether the relationship between dose and changes in behaviour operated through the intervention content. The indirect effects of content on changes in behaviour were not significant, indicating that intervention content was not a mediator of the relationship between dose and behaviour. However, we were likely underpowered to detect a significant effect as the total sample size in the mediation model was 25 clients.

Measures of perceived quality. The 20 clients who completed the Client Reflection had positive perceptions of the information and resources provided (all $M \mathrm{~s} \geq 6.00$ out of 7 ; see table 5). Clients' ratings of credibility were significantly related to changes in aerobic MVPA, as well as total session duration, total number of sessions, and number of times behavioural strategies were discussed $(p \mathrm{~s}<.05)$. Thus, clients who felt the counselor and service were highly credible: (1) reported the greatest increases in their aerobic MVPA over the service, (2) had the greatest exposure to the counselor (intervention dose), and (3) discussed behavioural strategies more frequently. No other correlations were significant. See table 5 for complete correlational results. 
Clients' perception of the personal importance of the content discussed during counseling sessions was significantly related to total session duration, total number of sessions, and number of times behavioural strategies were discussed over the 6-month service $(p s<.01)$. We ran a series of hierarchical linear regressions to test whether changes in intentions and behaviour could be predicted by the significantly correlated variables of dose, content and perceived quality. Due to issues of multicollinearity between the predictor variables, neither stepwise nor enter methods of regression allowed for further examination of the predictors of changes in intentions and MVPA.

\section{Discussion}

In line with Graham and colleagues' KTA framework [10], this study incorporated monitoring of intervention use to explore the relationship between implementation and changes in clients' LTPA intentions and behaviour during the second phase of the GIM service (September 2011 to January 2014). As hypothesized, clients had strong intentions for engaging in LTPA when they enrolled in the service that were sustained over their participation in the service. Further, clients' self-reported time spent in strength-training and total MVPA increased during the first two months and was sustained over months 2 to 6 of the service. While changes in clients' aerobic MVPA did not achieve statistical significance, a small effect size was seen over the first two months. Furthermore, changes in aerobic MVPA were significantly positively related to several measures of intervention dose, content, and perceived quality.

\section{LTPA Intentions and Behaviour}

Our hypothesis with respect to clients' LTPA intentions was confirmed - clients reported strong intentions for engaging in LTPA at baseline, and these strong intentions were sustained 
throughout their participation in the service. Given how the GIM service was promoted (i.e., websites, events, newsletters, and word of mouth), only clients who were interested in gaining support for LTPA participation would have contacted the GIM Program Coordinator; thus, it is not particularly surprising that clients reported strong LTPA intentions when they enrolled. The maintenance of these positive LTPA intentions over their enrolment is in line with both previous experimental findings of LTPA telephone counseling in the SCI population [20], as well as the previous evaluation of the first phase of the GIM service [26], suggesting that telephone-based counseling can sustain clients' motivation for engaging in LTPA under real-world conditions.

Our hypothesis concerning changes in LTPA behaviour was partially confirmed. Specifically, clients reported significant increases in time spent in strength-training and total MVPA between baseline and 2 months, and baseline and 6 months. There were no significant changes in aerobic MVPA. The magnitude of change in total MVPA between baseline and 2 months $(d=.36)$ is comparable with the small to medium-sized increases observed in our previous RCTs within the SCI population ( $d \mathrm{~s}=.38$ and $.55 ;$ [20] and [19], respectively). These findings suggest that the GIM service is just as effective at increasing moderate-to-vigorous LTPA behaviour under real-world conditions as it is in controlled, experimental conditions.

The initial increase in clients' strength-training and total MVPA in the first two months of the service was maintained over months 2 to 6 . Interestingly, the majority of dropouts occurred during the first two months of the service. Other community-based, LTPA telephone counseling programs have also reported a high attrition during the early stages of the intervention (e.g., up to one third of participants drop-out after first counseling call; [46]). Together, these findings suggest that the first two months of counseling may be a critical period for eliciting behaviour change and minimizing attrition among GIM clients. These findings are in contrast to two systematic reviews of telephone-delivered LTPA and/or dietary behaviour change interventions 
across a range of populations that indicate that interventions of longer duration (i.e., delivered over a greater number of months) have greater effectiveness [30, 47]. The discrepancy in these findings may be due to the differences in the scope of the interventions and the populations targeted. The GIM service aims to increase a single behaviour (i.e., LTPA) rather than a variety of complex health behaviours (e.g., LTPA and dietary behaviours combined; [30, 47]), and it is easier for clients to focus on changing one behaviour at a time [48]. Also, individuals with SCI frequently experience a range of secondary complications (e.g., pressure sores, pain; [49]) that may hinder ongoing participation in LTPA above and beyond the barriers to LTPA commonly reported by the general population (e.g., weather, time; [50]). The longer the intervention duration, the more likely individuals with SCI may drop out because of the LTPA barriers they face; thus, in line with our findings, interventions implemented over fewer months may be more effective for this population. Future iterations of the GIM service should focus on maximizing client adherence and changes in LTPA behaviour during the first two months of the service.

Despite a lack of significant increases in clients' aerobic MVPA throughout the service, small effect sizes emerged. When clients' aerobic MVPA is compared to the Physical Activity Guideline recommendations for aerobic LTPA (i.e., moderate-to-vigorous aerobic LTPA twice per week = 40 minutes of aerobic MVPA/week; [12]), on average, clients went from not meeting these aerobic recommendations at baseline (i.e., mean of 38.10 mins/week) to surpassing the recommendations at 2 and 6 months (i.e., 63.04 and 51.04 minutes/week, respectively). Furthermore, secondary analysis of the aerobic MVPA data revealed that compared to the $19.0 \%$ of clients who met aerobic recommendations when they enrolled in GIM, the percentage of clients meeting aerobic recommendations was significantly greater at 2 months $(73.1 \%$, $\left.\operatorname{McNemar} X^{2}=11.267, p=.008\right)$ and 6 months $\left(52.0 \%\right.$, McNemar $\left.X^{2}=3.000, p=.05\right)$. Thus while not statistically significant, the average increases in clients' aerobic MVPA are of clinical 
significance, as a greater percentage of clients were engaging in amounts of aerobic MVPA that are associated with fitness benefits at the 2- and 6-month time points [2].

\section{Implementation Correlates of Change in LTPA Intentions and Behaviour}

Changes in aerobic MVPA between baseline and 6 months were significantly positively related to intervention dose. These findings align with reports of LTPA telephone counseling programs among the general population that have found that the completion of a higher number of calls is associated with greater increases in clients' LTPA [30]. Thus, the current study confirms that there is a positive relationship between counseling dose and increases in LTPA behaviour among the SCI population.

Upon examination of content delivered during the counseling sessions, the number of times that the counselor discussed both informational and behavioural strategies was related to changes in aerobic MVPA over the 6-month period. Rather than adhering to a set implementation protocol, the GIM counselor tailored counseling session content to reflect clients' individual LTPA interests, motivations, and/or barriers. Since the provision of LTPA information is highly desired among people with SCI [3, 9], it is not surprising that the GIM clients who frequently discussed informational strategies with the counselor were more likely to report increases in their LTPA behaviour. With regard to behavioural strategies, findings from our two previous RCTs ([19] and [20]) suggest that teaching behavioural skills (specifically action and coping planning) during telephone-based counseling leads to increases in LTPA behaviour among people with SCI. Thus, including both informational and behavioural strategies in telephone-based counseling appears conducive to increasing aerobic MVPA.

The only Client Reflection variable that emerged as a correlate of 6-month change in aerobic MVPA was clients' perceptions of the credibility of the content provided. Previous research has suggested that people with SCI want to receive LTPA information from a credible 
source, such as a trained exercise counselor [51,52]. Clients who felt they were receiving credible information from a credible source may have been more willing to adopt and apply this content, making them more likely to increase their LTPA levels (c.f., Diffusion of Innovations; [53]). However, it should be noted that the Client Reflection data were collected only from individuals who completed the 6-month service, and clients' perceptions of credibility may have impacted whether or not they chose to continue their participation in the service. As discussed above, clients who received a greater number of counseling sessions and had greater total session durations were more likely to report changes in their LTPA levels. In the future, administering the Client Reflection to clients who choose to discontinue their participation would enhance our understanding of how credibility may impact the effectiveness of such services.

\section{Strengths and Limitations}

This study demonstrates that the GIM service can effectively sustain clients' LTPA intentions and increase LTPA behaviour at a magnitude that is comparable to controlled, experimental findings [19, 20], extending the literature on translating LTPA-enhancing telephone-based interventions to practical settings [30]. This study also provides evidence that the service's effectiveness is robust across systematic replications of the service (i.e., first and second phases of GIM; [26]), and thus makes an important contribution to translational research on health-promoting interventions [54].

To our knowledge, this study is the first to examine the relationship between the implementation and effectiveness of a nationwide telephone-based LTPA counseling service. By rigorously monitoring implementation (i.e., with the Counseling Session Checklists), we were able to address the "monitoring knowledge use" stage in the action cycle of the KTA framework [10], thereby allowing us to explore the relationships between intervention dose, content, perceived quality and changes in clients' LTPA intentions and behaviour. This was not possible 
in our previous evaluation of the first phase of the GIM service [26]. It should be noted that, similar to Goode and Eakin's study [55], initial and ongoing collaboration between the service's staff and research team was critical for the successful implementation, monitoring, and evaluation of the program.

Another strength of the current study is the identification of implementation variables as correlates of LTPA behaviour change for an evidence- and theory-based LTPA telephone counseling service applied within the community. The identified key implementation variables (i.e., correlates of change) can be specifically targeted to enhance the effectiveness of future iterations of the service in the same or additional contexts [27, 56-58]. To date, the GIM service protocol manual has been shared with, and adopted by, two additional contexts (one in Quebec, Canada, one in Sweden); as such, the methodology and results from the current study will be shared with these organizations/researchers so that these other adopters are aware of how to monitor service implementation correlates of LTPA behaviour change. This study provides additional research evidence that will help these settings adapt and evaluate the GIM service in their local context, thereby initiating another action cycle and broadening our understanding of how to apply the KTA framework for the implementation of a real-world intervention across multiple settings.

Despite these strengths, several limitations to the study should be noted. First, LTPA behaviour data were self-reported by clients and collected by the interventionist. Self-reported LTPA participation is considered one of the only valid ways to assess LTPA levels among people with SCI [59]. Nevertheless, clients may have over-reported their LTPA levels at 2-, 4-, and 6months because they anticipated that the counselor would want them to have higher LTPA levels as they progress in the service. However, as noted in the Methods section, the collection of LTPA data by the counselor was strategic in order for the counselor to monitor clients' progress 
and adjust counseling to suit the clients' changing needs and LTPA levels. Further, because within-person change in behaviour was the outcome of interest, error in data collection (i.e., overreporting LTPA) should be consistent across individuals over the study period.

A second limitation was that the small sample size and high multicollinearity among the correlates of change left us underpowered to conduct mediational analyses to further investigate whether the implementation of a specific type of intervention strategy (information vs. behavioural) was a potential mechanism/mediator by which exposure to the service leads to changes in LTPA intentions and behaviour. The examination of mediating mechanisms between implementation and effectiveness of rehabilitation services is a suggested avenue for future research.

Finally, we were able to examine intervention dose, content, and perceived quality of the service as potential implementation variables that may influence changes in LTPA intentions and behaviour, but there are likely other implementation variables that influence GIM's effectiveness that were not examined in the current study. Nevertheless, as Meyers, Durlak, and Wandersman [60] have noted, rigorous designs that encompass all of the possible influential variables are impossible to execute when studying implementation in real-world contexts. Thus, this study begins to uncover some of the key correlates of the service's effectiveness by focusing on intervention dose, which has been shown to influence telephone-based intervention outcomes in the general population, as well as intervention content, which has been shown to influence theory-based determinants of LTPA participation among people with SCI. Future studies examining rehabilitation interventions should consider monitoring intervention dose, content, and other implementation variables (e.g., clients' perception of the personal importance of the content discussed) to better understand the influences on intervention effectiveness. 


\section{Conclusions}

As an evidence- and theory-based intervention that operates under real-world conditions, GIM is effective at sustaining LTPA intentions and increasing time spent in strength-training and total MVPA among clients. By carefully monitoring implementation, we found that measures of intervention dose, content, and perceived credibility were related to changes in clients' aerobic LTPA. Findings from this study suggest that the first two months of the service are a critical period for both enhancing LTPA participation among the SCI population and for minimizing attrition, and that the provision of both informational and behavioural strategies are important for increasing clients' aerobic LTPA. Overall, this study provides additional knowledge that will contribute to the refinement of GIM as it enters its third phase, and identifies key implementation variables for an LTPA telephone counseling service for adults with SCI.

\section{Acknowledgements}

The authors would like to acknowledge: SCI Ontario and SCI British Columbia for their assistance with promoting the GIM service; Clement Lau, Krystina Malakovski, Krystn Orr, Christine Palisoc, Maryam Somo, Jessie Stapleton, and Matthew Stork for their assistance with data collection and management; Bryce Donald for implementing the counseling sessions; and Brittany McEachern for her assistance with manuscript formatting.

\section{Declaration of interest}

Funding for the second phase of the GIM service was provided by the Rick Hansen Institute and the Ontario Neurotrauma Foundation. This work was supported by an Ontario Neurotrauma 
Foundation and Rick Hansen Institute Mentor-Trainee Capacity Building Award in Knowledge Mobilization awarded to JRT and KMG. ALC holds a Tier 2 Canada Research Chair in physical activity promotion and disability from the Canadian Institutes of Health Research. KAN, ALC, and KMG conducted the randomized controlled trials upon which the Get in Motion service is based, and all four authors conducted the evaluation of the first phase of the service. The authors alone are responsible for the content and writing of this article.

\section{References}

1. Godin G, Shepherd RJ. A simple method to assess exercise behavior in the community. Canadian Journal of Applied Sports Science. 1985;10:141-6.

2. Hicks AL, Martin Ginis KA, Pelletier C, Ditor DS, Foulon B, Wolfe DL. The effects of exercise training on physical capacity, strength, body composition, and functional performance among adults with spinal cord injury: a systematic review. Spinal Cord. 2011;49:1103-27.

3. Wolfe DL, Martin Ginis KA, Latimer AE, Foulon B, Eng JJ, Hicks AL, et al. Physical activity and SCI. In: Eng JJ, Teasell RW, Miller WC, Wolfe DL, Townsen AF, Hsieh JTC, editors. Spinal Cord Injury Rehabilitation Evidence (Version 30). 2010.

4. Martin Ginis KA, Jetha A, Mack DE, Hetz S. Physical activity and subjective well-being among people with spinal cord injury: a meta-analysis. Spinal Cord. 2010;48:65-72.

5. Boschen KA, Miller WC, Noreau L, Wolfe DL, McColl MA, Martin Ginis KA, et al. Chapter 4: Community reintegration following spinal cord injury. In: Eng J, Teasell W, Miller WC, editors. Spinal Cord Injury Rehabilitation Evidence (Version 20). Vancouver, British Columbia: ICORD; 2008.

6. Sweet SN, Martin Ginis KA, Tomasone JR. Investigating intermediary variables in the physical activity and quality of life relationship in persons with spinal cord injury. Health Psychol. 2013;32:877-85.

7. Tomasone JR, Wesch N, Martin Ginis KA, Noreau L. Spinal cord injury, physical activity, and quality of life: A systematic review. Kinesiol Rev. 2013;2:113-29.

8. Martin Ginis KA, Latimer AE, Arbour-Nicitopoulos KP, Buchholz AC, Bray SR, Craven $\mathrm{BC}$, et al. Leisure time physical activity in a population-based sample of people with spinal cord injury Part I: Demographic and injury-related correlates. Arch Phys Med Rehabil. 2010;91:722-8.

9. Rimmer JH, Riley B, Wang E, Rauworth A, Jurkowski J. Physical activity participation among persons with disabilities: barriers and facilitators. Am J Prev Med. 2004;26(5):41925 .

10. Graham ID, Logan J, Harrison MB, Strauss SE, Tetroe J, Caswell W, et al. Lost in knowledge translation: Time for a map? . J Contin Educ Health Prof. 2006;26:13-24. 
11. Martin Ginis KA, Latimer-Cheung AE, Corkum S, Ginis S, Anathaspoulos P, ArbourNicitopoulos KA, et al. A case study of a community-university multidisciplinary partnership approach to increasing physical activity participation among people with spinal cord injury. Transl Behav Med. 2012;2:516-22.

12. Martin Ginis KA, Hicks AL, Latimer AE, Warburton DER, Bourne C, Ditor DS, et al. The development of evidence-informed physical activity guidelines for adults with spinal cord injury. Spinal Cord. 2011;49:1088-96.

13. Arbour-Nicitopoulos KP, Martin Ginis KA, Latimer-Cheung AE, Bourne C, Campbell D, Cappe D, et al. Development of an evidence-informed leisure time physical activity resource for adults with spinal cord injury: the SCI Get Fit Toolkit. Spinal Cord. 2013;51:491-500.

14. Latimer-Cheung AE, Arbour-Nicitopoulos KA, Brawley LR, Gray C, Wilson AJ, Prapavessis H, et al. Developing physical activity interventions for adults with spinal cord injury. Part 2: Motivational counseling and peer-mediated interventions for people intending to be active. Rehabil Psychol. 2013;51:273-80.

15. Michie S, Johnston M, Francis J, Hardeman W, Eccles M. From theory to intervention: Mapping theoretically derived behavioral determinants to behavior change techniques. Applied Psychology: An International Review. 2008;57(4):660-80.

16. Kahn EB, Ramsay LT, Brownson RC, Heath GW, Howze EH, Powell KE, et al. The effectiveness of interventions to increase physical activity: A systematic review. Am J Prev Med. 2002;22:73-107.

17. Bassett RL, Martin Ginis KA. Risky business: The effects of an individualized health information intervention on health risk perceptions and leisure time physical activity among people with spinal cord injury. Disabil Health J. 2011;4:165-76.

18. Foulon BL, Martin Ginis KA, Benedict C, Latimer AE, Sinden AR. The effects of a single wheelchair sports session on physical activity cognitions and behavior. In: Mohiyeddini $\mathrm{C}$, editor. Advances in the Psychology of Sports and Exercise New York: Nova; 2013. p. 181-90.

19. Arbour-Nicitopoulos KA, Martin Ginis KA, Latimer AE. Planning, leisure-time physical activity, and coping self-efficacy in persons with spinal cord injury: a randomized controlled trial. Arch Phys Med Rehabil. 2009;90:2003-11.

20. Latimer AE, Martin Ginis KA, Arbour KA. The efficacy of an implementation intention intervention for promoting physical activity among individuals with spinal cord injury: a randomized controlled trial. Rehabil Psychol. 2006;51:273-80.

21. Brownson RC, Jones E. Bridging the gap: Translating research into policy and practice. Prev Med. 2009;49:313-5.

22. Owen N, Glanz K, Sallis JF, Kelder SH. Evidence-based approaches to dissemination and diffusion of physical activity interventions. Am J Prev Med. 2006;31(S35-S44).

23. Sallis JF, Owen N, Fotheringham MJ. Behavioral epidemiology: A systematic framework to classify phases of research on health promotion and disease prevention. Ann Behav Med. 2000;22:294-8.

24. Martin Ginis KA, Tomasone JR, Arbour-Nicitopoulos KA, Bassett-Gunter RL, Wolfe DL. Developing physical activity interventions for adults with spinal cord injury. Part 1: A comparison of social cognitions across actors, intenders, and non-intenders. Rehabil Psychol. 2013;58:299-306. 
25. Martin Ginis KA, Latimer AE, Arbour-Nicitopoulos KP, Bassett RL, Wolfe DL, Hanna SE. Determinants of physical activity among people with spinal cord injury: A test of social cognitive theory. Ann Behav Med. 2011;42:127-33.

26. Arbour-Nicitopoulos KA, Tomasone JR, Latimer-Cheung AE, Martin Ginis KA. Get In Motion: An evaluation of the reach and effectiveness of a physical activity telephone counseling service for Canadians living with spinal cord injury. Phys Med Rehabil. 2014;6:1088-96.

27. Durlak JA, DuPre EP. Implementation matters: A review of research on the influence of implementation on program outcomes and the factors affecting implementation. Am J Commun Psychol. 2008;41:327-50.

28. Dusenbury L, Brannigan R, Falco M, Hansen WB. A review of research on fidelity of implementation: Implications for drug abuse prevention in school settings. Health Educ Res. 2003;18:237-56.

29. Rabin BA, Brownson RC, Kerner JF, Glasgow RE. Methodologic challenges in disseminating evidence-based interventions to promote physical activity. Am J Prev Med. 2006;31:S24-S34.

30. Goode AD, Reeves MM, Eakin EG. Telephone-delivered interventions for physical activity and dietary behavior change: an updated systematic review. Am J Prev Med. 2012;42:81-8.

31. Goode AD, Winkler E, Lawler SP, Reeves MM, Owen N, Eakin EG. A telephonedelivered physical activity and dietary intervention for type 2 diabetes and hypertension: Does intervention dose influence outcomes? Am J Health Promot. 2011;25:257-63.

32. Greaves CJ, Sheppard KE, Abraham C, Hardeman W, Roden M, Evans PH, et al. Systematic review of reviews of intervention components associated with increased effectiveness in dietary and physical activity interventions. BMC Public Health. 2011;11:119.

33. Michie S, Abraham C, Whittington C, McAteer J, Gupta S. Effective techniques in healthy eating and physical activity interventions: A meta-regression. Health Psychol. 2009;28:690-701.

34. SCI Action Canada. SCI Action Canada Hamilton2012 [cited 2012 March 1]. Available from: http://sciactioncanada.ca/.

35. Canadian Paraplegic Association. The Workforce Participation Survey of Canadians with Spinal Cord Injury. Ottawa: Canadian Paraplegic Association, 2000.

36. Farry A, Baxter D. The incidence and prevalence of spinal cord injury in Canada: overview and estimates based on current evidence. Vancouver, BC: Rick Hansen Institute, 2010.

37. Rick Hansen Institute. Canadian Spinal Cord Injury Community Survey: Participant Reports - Traumatic Injuries. Vancouver: Rick Hansen Institute; 2011.

38. Martin Ginis KA, Latimer AE, Hicks AL, Craven BC. Development and preliminary evaluation of an activity measure for people with spinal cord injury. Med Sci Sports Exerc. 2005;37:1099-111.

39. Brawley LR, Arbour-Nicitopoulos KP, Martin Ginis KA. Developing physical activity interventions for adults with spinal cord injury. Part 3: A pilot feasibility study of an intervention to increase self-managed physical activity. Rehabil Psychol. 2013;58:316-21.

40. Latimer AE, Martin Ginis KA. The theory of planned behavior in prediction of leisure time physical activity among individuals with spinal cord injury. Rehabil Psychol. 2005;50:389-96. 
41. Martin Ginis KA, Latimer AE. The Leisure-Time Physical Activity Questionnaire for People with Spinal Cord Injury (LTPAQ-SCI) 2007. Available from: 10.1037/a0032816.

42. Martin Ginis KA, Phang SH, Latimer AE, Arbour-Nicitopoulos KA. Reliability and validity tests of the leisure time physical activity questionnaire for people with spinal cord injury. Arch Phys Med Rehabil. 2012;93:677-82.

43. Tomasone JR, Martin Ginis KA, Estabrooks PA, Domenicucci L. Changing Minds, Changing Lives from the top down: An investigation of the dissemination and adoption of a nationwide educational intervention to enhance health care professionals' intentions to prescribe physical activity. Int J Behav Med. 2015; 22:336-44. doi: 10.1007/s12529-0149414-6..

44. Tabachnick BG, Fidell LS. Using Multivariate Statistics, 5th Edition. Boston: Allyn \& Bacon; 2007.

45. Cohen J. A power primer. Quant Methods Psychol. 1992;112:155-9.

46. Goode AD, Reeves MM, Owen N, Eakin EG. Results from the dissemination of an evidence-based telephone-delivered intervention for healthy lifestyle and weight loss: the Optimal Health Program. Transl Behav Med. 2013;3(340-350).

47. Eakin EG, Lawler SP, Vandelanotte C, Owen N. Telephone interventions for physical activity and dietary behavior change: a systematic review. Am J Prev Med. 2007;32:41934.

48. Medical Research Council. Developing and evaluating complex interventions: New guidance 2008 [cited 201110 Feb 2011]. Available from:

http://www.mrc.ac.uk/complexinterventionsguidance.

49. Anson CA, Shepherd C. Incidence of secondary complications in spinal cord injury. Int J Rehabil Res. 1996;19:55-66.

50. Salmon J, Crawford D, Owen N, Bauman A, Sallis JF. Physical activity and sedentary behavior: A population-based study of barriers, enjoyment, and preference. Health Psychol. 2003;22:178-88.

51. Faulkner G, Gorczynski P, Arbour KP, Letts L, Wolfe DL, Martin Ginis KA. Messengers and methods of disseminating health information among individuals with spinal cord injury. In: Berkovsky TC, editor. Handbook of Spinal Cord Injuries. New York: Nova Science Publishers, Inc.; 2010. p. 329-74.

52. Letts L, Martin Ginis KA, Faulkner G, Colquhoun H, Levac D, Gorczynski P. Preferred methods and messengers for delivering physical activity information to people with spinal cord injury: A focus group study. Rehabil Psychol. 2011;56(2):128-37.

53. Rogers E. Diffusion of Innovations. 5th Edition ed. New York: Free Press; 2003.

54. Kessler R, Glasgow RE. A proposal to speed translation of healthcare research into practice: Dramatic change is needed. Am J Prev Med. 2011;40:637-44.

55. Goode AD, Eakin EG. Dissemination of an evidence-based telephone-delivered lifestyle intervention: factors associated with successful implementation and evaluation. Transl Behav Med. 2013;3:351-6.

56. Bonetti D, Eccles M, Johnston M, Steen N, Grimshaw J, Baker R, et al. Guiding the design and selection of interventions to influence the implementation of evidence-based practice: An experimental simulation of a complex intervention trial. Soc Sci Med. 2005;60:2135-47.

57. Tomasone JR, Martin Ginis KA, Estabrooks PA, Domenicucci L. "Changing Minds": Determining the effectiveness and key ingredients of an educational intervention to 
enhance health care professionals' intentions to prescribe physical activity to patients with physical disabilities. Implement Sci. 2014;9:30.

58. Wandersman A, Duffy J, Flaspohler P, Noonan R, Lubell K, Stillman L, et al. Bridging the gap between prevention research and practice: The Interactive Systems Framework for dissemination and implementation. Am J Commun Psychol. 2008;41:171-81.

59. Tanhoffer RA, Tanhoffer AIP, Raymond J, Hills AP, Davis GM. Comparison of methods to assess energy expenditure and physical activity in people with spinal cord injury. $\mathrm{J}$ Spinal Cord Med. 2012;35:35-45.

60. Meyers DC, Durlak JA, Wandersman A. The Quality Implementation Framework: A synthesis of critical steps in the implementation process. Am J Commun Psychol. 2012;50:462-80.

\section{Figure caption}

Figure 1. Client enrollment and participation in the Get in Motion service between September 2011 and January 2014, and amount of data available for the clients throughout the service.

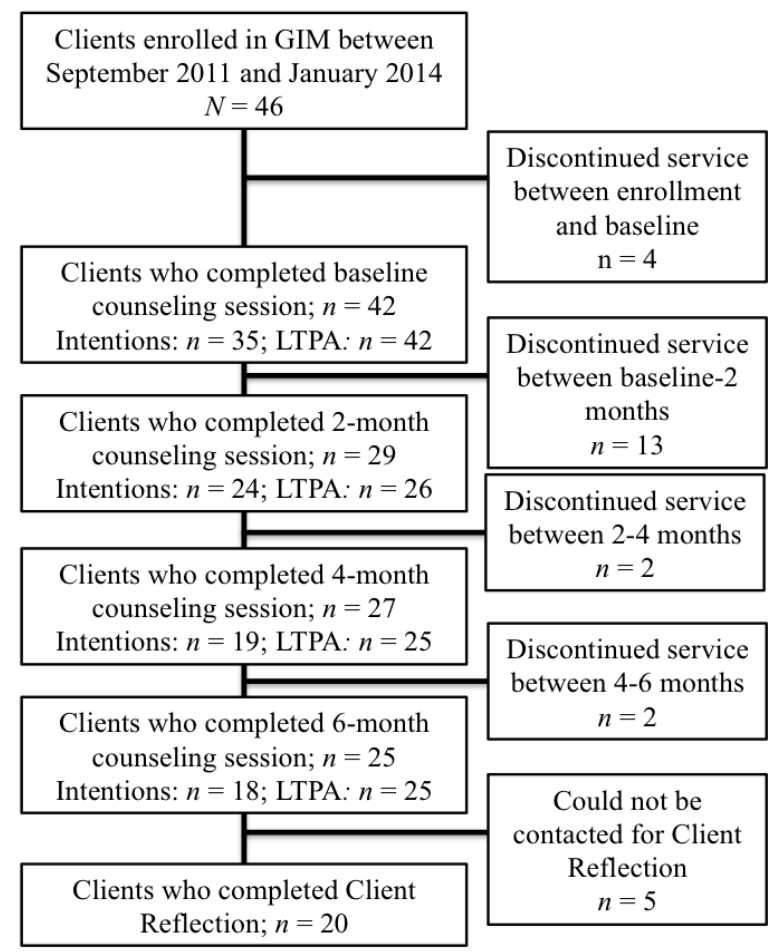

Note. GIM, Get in Motion; LTPA, leisure-time physical activity. Of the 13 clients who discontinued participation between baseline and 2 months, three could not be contacted by the counselor. The remaining 10 clients provided the following reasons for discontinuing: moved to a different country $(n=3)$, more interested in local/face-to-face resources $(n=3)$, personal situation $(n=2)$, and health complications not related to LTPA participation $(n=2)$. Of the four clients who discontinued between months 2 to 6 , two could not be contacted by the counselor, and the other two experienced health complications not related to LTPA participation. 
Table 1.

Demographic characteristics of the clients who enrolled in Get in Motion between September 2011 and January $2014(N=46)$ and comparison to those who discontinued $(n=21)$ versus completed $(n=25)$ their 6-months in the service.

\begin{tabular}{|c|c|c|c|}
\hline $\begin{array}{l}\text { Variable } \\
(n, \%)\end{array}$ & Enrolled & Discontinued & Completed \\
\hline & $n=46$ & $n=21$ & $n=25$ \\
\hline Age (years), $M(S D)$ & $51.46(\mathrm{SD} 12.36)$ & $49.90($ SD 10.72) & 52.50 (SD 13.89) \\
\hline Years post-injury, $M(S D)$ & $17.00(\mathrm{SD} 17.59$ & $15.25(\mathrm{SD} 17.18)$ & 17.71 (SD 18.19) \\
\hline \multicolumn{4}{|l|}{ Sex } \\
\hline Male & $23(50.0)$ & $13(61.9)$ & $10(40.0)$ \\
\hline Female & $22(47.8)$ & $8(38.1)$ & $14(56.0)$ \\
\hline \multicolumn{4}{|l|}{ Level of injury } \\
\hline Paraplegia & $23(50.0)$ & $9(42.9)$ & $14(56.0)$ \\
\hline Tetraplegia & $21(45.7)$ & $11(52.4)$ & $10(40.0)$ \\
\hline \multicolumn{4}{|l|}{ Cause of injury } \\
\hline Vehicular & $12(26.1)$ & $4(19.0)$ & $7(28.0)$ \\
\hline Sports/recreation/falls & $13(28.2)$ & $5(23.8)$ & $8(32.0)$ \\
\hline $\begin{array}{l}\text { Medical/surgical } \\
\text { complications }\end{array}$ & $8(17.4)$ & $3(38.1)$ & $5(20.0)$ \\
\hline Other & $13(28.2)$ & $8(38.1)$ & $5(20.0)$ \\
\hline \multicolumn{4}{|l|}{ Primary mode of mobility } \\
\hline Manual chair & $13(28.3)$ & $5(23.8)$ & $8(32.0)$ \\
\hline Power chair & $23(50.0)$ & $11(52.4)$ & $12(48.0)$ \\
\hline $\begin{array}{l}\text { Gait aid/Walk } \\
\text { independently }\end{array}$ & $10(21.7)$ & $5(23.8)$ & $5(20.0)$ \\
\hline \multicolumn{4}{|l|}{ Marital status } \\
\hline Single & $14(30.4)$ & $8(38.1)$ & $6(24.0)$ \\
\hline Married/common law & $24(52.2)$ & $12(57.1)$ & $12(48.0)$ \\
\hline $\begin{array}{l}\text { Divorced/widowed/ } \\
\text { separated }\end{array}$ & $8(17.3)$ & $1(4.8)$ & $7(28.0)$ \\
\hline \multicolumn{4}{|l|}{ Highest level of education } \\
\hline Post-secondary & $31(67.4)$ & $13(61.9)$ & $18(72.0)$ \\
\hline High school/other & $15(32.6)$ & $7(38.1)$ & $7(28.0)$ \\
\hline \multicolumn{4}{|l|}{ Geographic location } \\
\hline Western Canada & $19(67.4)$ & $9(42.9)$ & $10(40.0)$ \\
\hline Central Canada & $25(54.3)$ & $11(52.4)$ & $14(56.0)$ \\
\hline Eastern Canada & $2(4.3)$ & $1(4.8)$ & $1(4.0)$ \\
\hline
\end{tabular}

Note. All values are given as $n(\%)$ except age and years post injury, which are $M(S D)$, as indicated. $n \mathrm{~s}<46,21$, and 25 for some variables in the second, third, and fourth columns, respectively, due to non-responding.

There were no significant differences between the 21 dropouts and 25 completers for any demographic variable (all $p \mathrm{~s} \geq .13$ ). 
Table 2.

Informational and behavioural strategies used during Get in Motion counseling sessions.

\begin{tabular}{cc}
\hline Type of strategy & How strategy is used in the GIM service \\
\hline $\begin{array}{c}\text { Informational strategies } \\
\text { Physical Activity Guidelines for } \\
\text { Adults with SCI }\end{array}$ & $\begin{array}{c}\text { Counselor provides recommendations for how } \\
\text { much, how hard, and how often to engage in } \\
\text { aerobic and strength-training LTPA to achieve } \\
\text { fitness benefits. } \\
\text { Intensity classification }\end{array}$ \\
$\begin{array}{c}\text { Counselor describes how to determine whether } \\
\text { they are working at a mild, moderate, or heavy }\end{array}$ \\
intensity depending on how their body feels. \\
Counselor talks about the benefits of warming up \\
before and cooling down after exercising. \\
Counselor answers questions about whether \\
specific activities are classified as aerobic or \\
strength-training LTPA. \\
Counselor specifies websites or local facilities that \\
may be useful to clients' LTPA needs, \\
interests, and preferences. \\
Behavioural strategies \\
Goal-setting
\end{tabular}

Note. GIM, Get in Motion; LTPA; leisure-time physical activity; SCI, spinal cord injury.

The following studies refer to either the original references for the intervention strategies or the use of the given intervention strategies among the SCI population: ${ }^{\wedge}[12] ;{ }^{\wedge}[40] ;{ }^{\wedge \wedge}[19,20] ;^{\wedge}$

[19]. 
Table 3.

Clients' changes in LTPA intentions and behaviour over enrollment in the Get in Motion service.

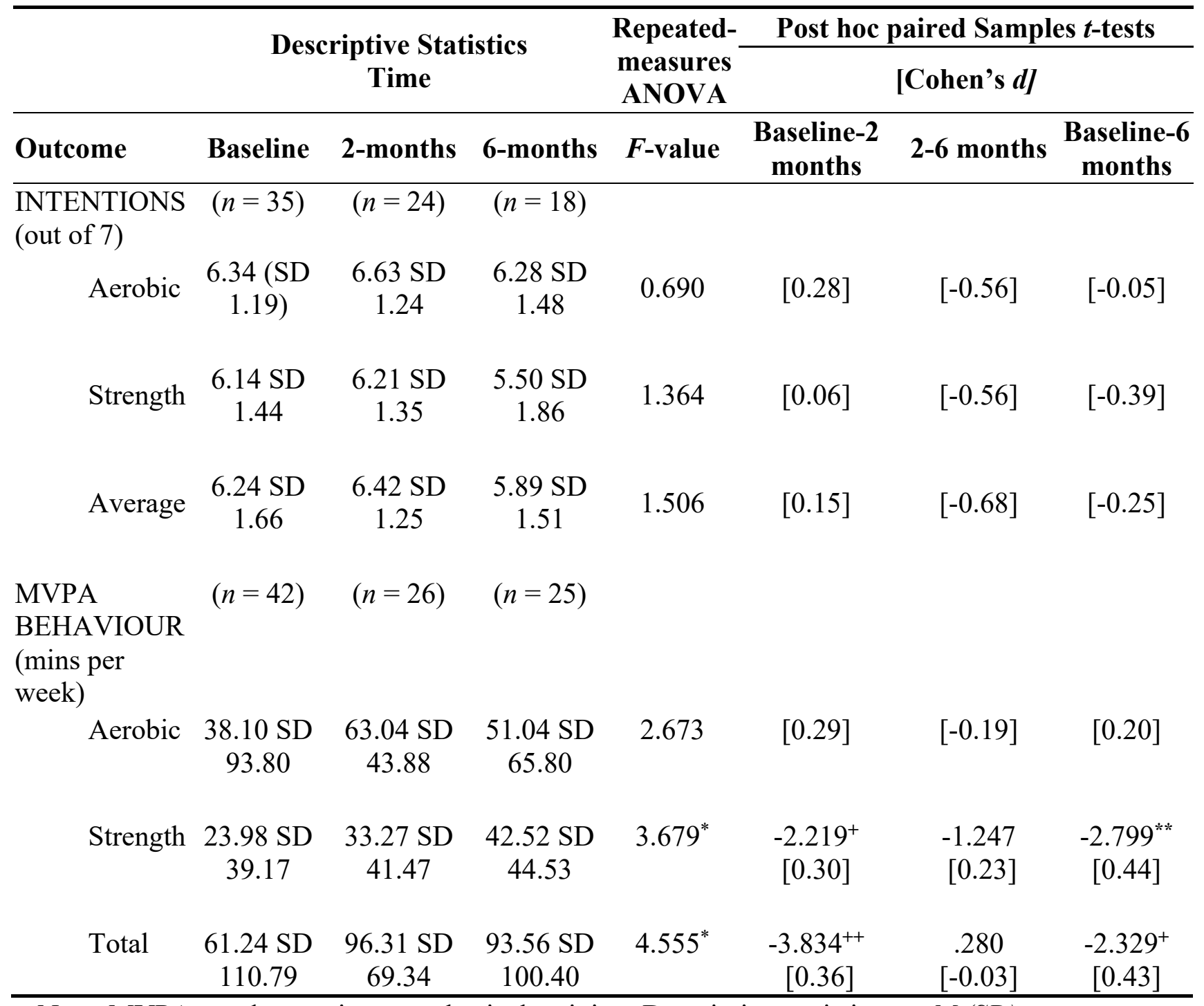

Note. MVPA, moderate-vigorous physical activity. Descriptive statistics are $M(S D)$, as indicated. Post-hoc paired samples $t$-tests were not examined for the repeated-measures ANOVAs that were not significant; hence, there are no $t$-values in those cells.

${ }^{*} p \leq .05,{ }^{+} p \leq .017$ (Bonferroni correction for multiple comparisons applied to t-tests), ${ }^{* *} p \leq .01$, ${ }^{++} p \leq .001$. 
Table 4.

Descriptive statistics for implementation dose and content between baseline to 2 months and 2 to 6 months of telephone counselling.

\begin{tabular}{|c|c|c|c|}
\hline $\begin{array}{l}\text { Implementation } \\
\text { variable }\end{array}$ & $\begin{array}{c}\text { Baseline to } 2 \text { months } \\
\text { M (SD) } \\
\text { (range) } \\
n=29\end{array}$ & $\begin{array}{c}2 \text { to } 6 \text { months } \\
\text { M (SD) } \\
\text { (range) } \\
n=25\end{array}$ & $\begin{array}{c}t \text {-value } \\
(p \text {-value })\end{array}$ \\
\hline \multicolumn{4}{|l|}{ Sessions } \\
\hline Number & $\begin{array}{c}3.18 \text { (SD 1.98) } \\
(0-7)\end{array}$ & $\begin{array}{c}2.76 \text { (SD 2.47) } \\
(0-7)\end{array}$ & $\begin{array}{l}1.420 \\
(.16)\end{array}$ \\
\hline $\begin{array}{l}\text { Total duration } \\
\text { (min) }\end{array}$ & $\begin{array}{c}58.39(\text { SD 36.98) } \\
(0-146.00)\end{array}$ & $\begin{array}{c}36.26 \text { (SD 35.93) } \\
(0-97.00)\end{array}$ & $\begin{array}{c}4.700 \\
(<.001)^{++}\end{array}$ \\
\hline \multicolumn{4}{|l|}{$\begin{array}{l}\text { Informational } \\
\text { strategies }\end{array}$} \\
\hline Number & $\begin{array}{c}7.35 \text { (SD 6.88) } \\
(0-37)\end{array}$ & $\begin{array}{c}4.85(\mathrm{SD} 4.85) \\
(0-14)\end{array}$ & $\begin{array}{l}2.487 \\
(.02)^{*}\end{array}$ \\
\hline Duration (min) & $\begin{array}{c}17.31(\text { SD 17.33) } \\
(0-63.80)\end{array}$ & $\begin{array}{c}10.53(\text { SD 11.88) } \\
(0-36.70)\end{array}$ & $\begin{array}{c}3.306 \\
(.002)^{* *}\end{array}$ \\
\hline \multicolumn{4}{|l|}{$\begin{array}{l}\text { Behavioural } \\
\text { strategies }\end{array}$} \\
\hline Number & $\begin{array}{c}5.96(\text { SD 4.09) } \\
(0-15)\end{array}$ & $\begin{array}{c}3.50(\text { SD 3.43) } \\
(0-11)\end{array}$ & $\begin{array}{c}4.848 \\
(<.001)^{++}\end{array}$ \\
\hline Duration (min) & $\begin{array}{c}17.50(\text { SD 14.91) } \\
(0-47.30)\end{array}$ & $\begin{array}{c}10.28 \text { (SD 11.35) } \\
(0-40.50)\end{array}$ & $\begin{array}{c}4.149 \\
(<.001)^{++}\end{array}$ \\
\hline
\end{tabular}

Note. Durations are in minutes. Degrees of freedom for $t$-tests were 45 .

${ }^{*} p \leq .05,{ }^{* *} p \leq .01,{ }^{++} p \leq .001$. 
Table 5.

Descriptive statistics and correlations between changes in behaviour and implementation dose, content, and perceived quality between baseline and 6 months.

\begin{tabular}{|c|c|c|c|c|c|c|c|c|c|c|c|c|c|c|}
\hline Variable & $\begin{array}{l}\mathrm{M}(\mathrm{SD}) \\
\text { [range] }\end{array}$ & 1 & 2 & 3 & 4 & 5 & 6 & 7 & 8 & 9 & 10 & 11 & 12 & $13 \quad 14$ \\
\hline $\begin{array}{l}\text { 1. } \triangle \text { Aerobic } \\
\text { MVPA }\end{array}$ & $\begin{array}{c}10.48(\mathrm{SD} \\
64.96)\end{array}$ & - & & & & & & & & & & & & \\
\hline 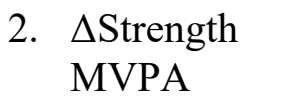 & $\begin{array}{c}22.84(\mathrm{SD} \\
40.79)\end{array}$ & -.145 & -- & & & & & & & & & & & \\
\hline 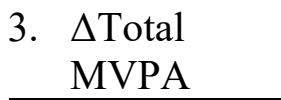 & $\begin{array}{c}33.32(\mathrm{SD} \\
71.52)\end{array}$ & $\begin{array}{c}.826 \\
++\end{array}$ & $\begin{array}{c}.439 \\
*\end{array}$ & -- & & & & & & & & & & \\
\hline $\begin{array}{l}\text { 4. Total } \\
\text { duration }\end{array}$ & $\begin{array}{c}103.11(\mathrm{SD} \\
71.66) \\
{[0-248]}\end{array}$ & $\begin{array}{c}.479 \\
*\end{array}$ & -.215 & .312 & -- & & & & & & & & & \\
\hline $\begin{array}{l}\text { 5. Total } \\
\text { sessions }\end{array}$ & $\begin{array}{c}5.93(\mathrm{SD} 4.01) \\
{[0-13]}\end{array}$ & $\begin{array}{l}.534 \\
* *\end{array}$ & -.278 & .327 & $\begin{array}{c}.925 \\
++\end{array}$ & -- & & & & & & & & \\
\hline $\begin{array}{l}\text { 6. Info } \\
\text { duration }\end{array}$ & $\begin{array}{c}29.99(\mathrm{SD} \\
27.99) \\
{[0-103.60]}\end{array}$ & .222 & -.188 & .095 & $\begin{array}{l}.775 \\
++\end{array}$ & $\begin{array}{l}.782 \\
* *\end{array}$ & -- & & & & & & & \\
\hline 7. Info times & $\begin{array}{c}13.63(\mathrm{SD} \\
10.83) \\
{[0-41]}\end{array}$ & $\begin{array}{c}.398 \\
*\end{array}$ & -.295 & .193 & $\begin{array}{c}.765 \\
++\end{array}$ & $\begin{array}{c}.841 \\
++\end{array}$ & $\begin{array}{c}.824 \\
++\end{array}$ & -- & & & & & & \\
\hline $\begin{array}{l}\text { 8. Behav } \\
\text { duration }\end{array}$ & $\begin{array}{c}29.34((\mathrm{SD} \\
24.81) \\
{[0-81.34]}\end{array}$ & .226 & -.156 & .116 & $\begin{array}{l}.848 \\
* *\end{array}$ & $\begin{array}{c}.873 \\
++\end{array}$ & $\begin{array}{l}.803 \\
* *\end{array}$ & $\begin{array}{c}.819 \\
++\end{array}$ & -- & & & & & \\
\hline $\begin{array}{l}\text { 9. Behav } \\
\text { times }\end{array}$ & $\begin{array}{c}10.17(\mathrm{SD} 7.16) \\
{[0-25]}\end{array}$ & $\begin{array}{c}.458 \\
*\end{array}$ & -.141 & .336 & $\begin{array}{c}.895 \\
++\end{array}$ & $\begin{array}{c}.688 \\
++\end{array}$ & $\begin{array}{c}.524 \\
*\end{array}$ & $\begin{array}{c}.789 \\
++\end{array}$ & $\begin{array}{c}.874 \\
++\end{array}$ & - & & & & \\
\hline $\begin{array}{l}\text { 10. New } \\
\text { information }\end{array}$ & $\begin{array}{c}6.00(\mathrm{SD} 1.59) \\
{[2-7]}\end{array}$ & .135 & -.251 & -.008 & -.103 & -.243 & -.282 & .114 & -.184 & -.006 & -- & & & \\
\hline 11. Interesting & $\begin{array}{c}6.50(\mathrm{SD} 0.69) \\
{[5-7]}\end{array}$ & .417 & -.366 & .189 & .130 & -.047 & -.080 & .321 & .066 & .234 & $\begin{array}{c}.724 \\
++\end{array}$ & -- & & \\
\hline
\end{tabular}




\begin{tabular}{|c|c|c|c|c|c|c|c|c|c|c|c|c|c|c|c|}
\hline $\begin{array}{l}\text { 12. Easy to } \\
\text { understand }\end{array}$ & $\begin{array}{c}6.55 \text { (SD 0.76) } \\
{[4-7]}\end{array}$ & .116 & -.137 & .035 & .242 & .148 & .145 & .227 & .333 & .306 & .010 & -.106 & -- & & \\
\hline 13. Credible & $\begin{array}{c}6.65 \text { (SD 0.49) } \\
{[6-7]}\end{array}$ & $\begin{array}{c}.532 \\
*\end{array}$ & -.543 & .202 & $\begin{array}{c}.462 \\
*\end{array}$ & $\begin{array}{l}.574 \\
* *\end{array}$ & .394 & .271 & .370 & $\begin{array}{c}.447 \\
*\end{array}$ & .271 & $.447^{*}$ & -.021 & -- & \\
\hline $\begin{array}{l}\text { 14. Personally } \\
\text { important }\end{array}$ & $\begin{array}{c}6.60 \text { (SD 0.68) } \\
{[5-7]}\end{array}$ & .414 & -.030 & .360 & $\begin{array}{l}.565 \\
* *\end{array}$ & $\begin{array}{c}.679 \\
++\end{array}$ & .372 & .377 & .351 & $\begin{array}{l}.570 \\
* *\end{array}$ & .389 & .383 & .244 & $\begin{array}{c}.506 \\
*\end{array}$ & -- \\
\hline
\end{tabular}

Note. $\Delta$, change in type of MVPA over 6 months in (minutes/week); MVPA, moderate-vigorous physical activity. Items 4-9 represent the six variables of implementation dose and content extracted from the Counseling Session Checklists. "Info" represents

informational strategies and "Behav" represents behavioural strategies discussed during counseling sessions. Durations are in minutes. Ranges for implementation variables are indicated in brackets. Items 10-14 represent clients' perceived quality of implementation (five items from the Client Reflection) and are scored out of 7.

$* \mathrm{p}<.05, * * \mathrm{p}<.01,{ }^{++} \mathrm{p}<.001$ 
